\title{
Difluoroalkylation of tertiary amides and lactams by an iridium- catalyzed reductive Reformatsky reaction
}

\author{
Phillip Biallas, Ken Yamazaki, and Darren J. Dixon*[a] \\ [a] Dr. P. Biallas, K. Yamazaki, Prof. Dr. D. J. Dixon \\ Chemistry Research Laboratory, Department of Chemistry \\ University of Oxford \\ 12 Mansfield Road, Oxford (UK) \\ E-mail: darren.dixon@chem.ox.ac.uk \\ Supporting information for this article is given via a link at the end of the document.
}

\begin{abstract}
An iridium catalyzed, reductive alkylation of abundant tertiary lactams and amides with difluoro-Reformatsky reagents $\left(\mathrm{BrZnCF}_{2} \mathrm{R}\right)$ for the general synthesis of medicinally relevant $\alpha$ difluoroalkylated tertiary amines, is described. Operating under mild conditions using 1-2 mol\% of Vaska's complex $\left(\mathrm{IrCl}(\mathrm{CO})\left(\mathrm{PPh}_{3}\right)_{2}\right)$ and tetramethyldisiloxane (TMDS), chemoselective reductive generation of the $O$-silylated hemiaminals provided unique access to the key reactive iminium ion intermediates, facilitating efficient downstream carbon-carbon bond formation. A broad scope (42 examples), including $\mathrm{N}$-aryl and $\mathrm{N}$-heteroaryl substituted lactams, demonstrated an excellent functional group tolerance, which was further highlighted by the late-stage functionalization of four drug molecules. A gram scale synthesis and common downstream transformations to a variety of $\mathrm{CF}_{2}$-containing compounds proved the potential synthetic relevance of this new methodology in medicinal chemistry.
\end{abstract}

The incorporation of the gem-difluoromethylene $\left(-\mathrm{CF}_{2^{-}}\right)$ group, an oxygen bioisostere, ${ }^{[1]}$ into organic molecules has gained considerable attention in pharmaceutical and agrochemical research, as well as in materials science, due to the unique influence of fluorine atoms on physical, chemical and biological properties. ${ }^{[2]}$ More specifically, the $\beta, \beta$-difluoro- $\alpha$-amino motif represents a key building block in many bioactive molecules, owing to the electronic influence of the fluorine atoms on the neighboring nitrogen center. The strong electron-withdrawing character of $\beta$-fluorine substitution on amines or nitrogencontaining heterocycles significantly lowers their basicity and $\mathrm{p} K_{\mathrm{a}}$, which in turn influence critical parameters in medicinal lead optimization, such as physicochemical properties, binding affinities and absorption, distribution, metabolism, excretion (ADME). ${ }^{[3]}$ The relevance of this structural motif in drug discovery is further exemplified by the large variety of $\beta, \beta$-difluoro- $\alpha$-aminocontaining pharmaceutical compounds such as gemcitabine, ${ }^{[4]}$ cedazuridine, ${ }^{[5]}$ eflornithine, ${ }^{[6]}$ GDC-0077 ${ }^{[7]}$ and glecaprevir ${ }^{[8]}$ (Scheme 1A). Therefore, the development of new concise and selective methods for the late-stage introduction of $\mathrm{gem}$ difluoromethylene units onto nitrogen-containing scaffolds remains an attractive goal in synthetic chemistry. ${ }^{[9]}$

In the past decade, several research groups have become involved in the challenging late-stage reductive $\mathrm{C}-\mathrm{C}$ coupling of amides with organometallic reagents for the synthesis of $\alpha$ functionalized amines. ${ }^{[10]}$ Stoichiometric approaches for the reductive functionalization of different amide classes, including lactams, with various organometallic reagents have been reported by Huang, ${ }^{[11]}$ Sato and Chida, ${ }^{[12]}$ Chiba and our group. ${ }^{[13]}$ These methods employ DIBAL-H, Schwartz's reagent $\left(\mathrm{Cp}_{2} \mathrm{ZrHCl}\right)$, triflic anhydride/metal hydride or a $\mathrm{NaH} / \mathrm{Nal}$ composite as the stoichiometric reductants. A highly chemoselective reductive functionalization of amides can be achieved by a transition metal-catalyzed approach, as demonstrated by our group ${ }^{[14]}$ and others. ${ }^{[15]}$ Using catalytic amounts of Vaska's complex $\left(\operatorname{IrCl}(\mathrm{CO})\left(\mathrm{PPh}_{3}\right)_{2}\right)$ and 1,1,3,3tetramethyldisiloxane (TMDS) led to the formation of meta-stable $O$-silylated hemiaminal intermediates, which are precursors to

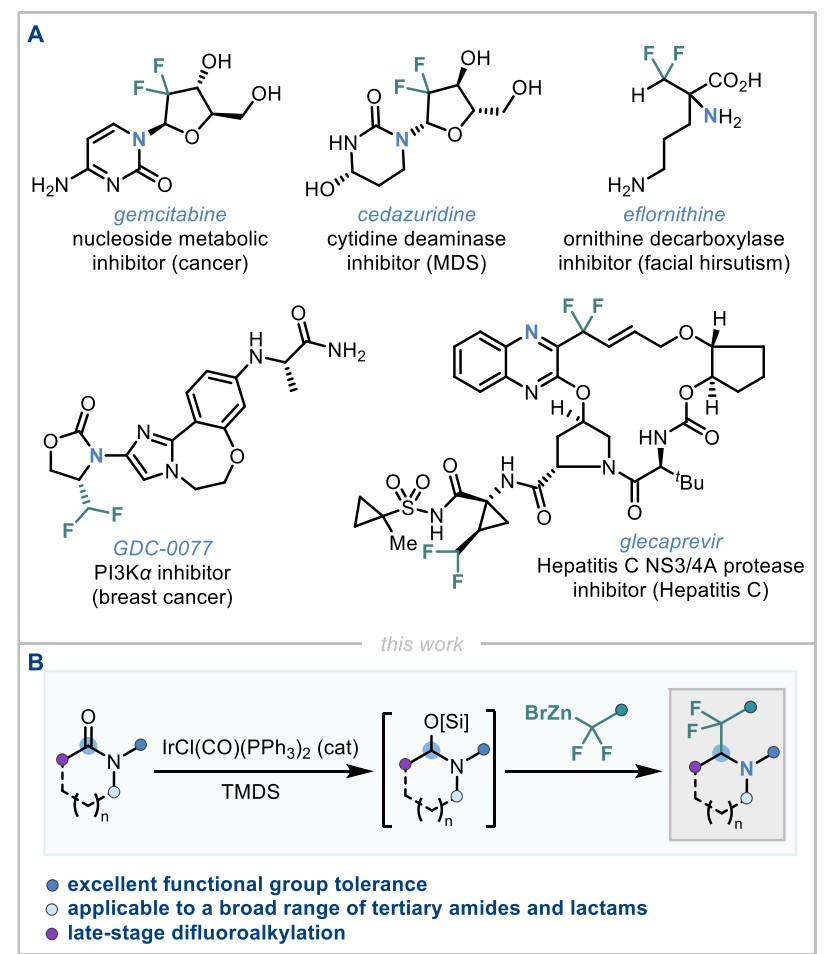

Scheme 1. A) Drug molecules containing the gem-difluoro motif; B) Reductive functionalization of amides and lactams by an iridium-catalyzed Reformatsky reaction.

reactive iminium ions that can undergo subsequent nucleophilic functionalization.

Continuing our group's ongoing efforts on reductive iridiumcatalyzed $\mathrm{C}-\mathrm{C}$ bond forming reactions, we envisioned combining amide functionalization with commonly-known difluoromethylene sources to form highly desirable and medicinally relevant $\alpha$ difluoroalkylated amines (Scheme 1B). The ethoxycarbonyldifluoromethyl $\left(-\mathrm{CF}_{2} \mathrm{CO}_{2} \mathrm{Et}\right)$ moiety is a versatile difluoromethylene source, due to its potential as a handle for further modifications into various functional groups. ${ }^{[16]}$ In addition to cross-coupling, ${ }^{[17]} \mathrm{C}-\mathrm{H}$ functionalization ${ }^{[16 i, 17 a, 18]}$ and radical 
addition $^{[18 a, 19]}$, this difluoro-methylene containing unit is traditionally introduced via nucleophilic attack of the corresponding difluoro-Reformatsky reagent $\left(\mathrm{BrZnCF}_{2} \mathrm{CO}_{2} \mathrm{Et}\right)$ on carbonyl groups, imines or azodicarboxylates. ${ }^{20]}$ This longserving reagent with its efficacious reactivity towards various electrophiles caught our attention for its potential unprecedented deployment in a general late-stage amide functionalization approach and herein, we wish to report our findings.

$N, N$-Dimethyl-1-naphthamide 1a was chosen as a model substrate for the reductive functionalization with difluoroorganozinc reagent $\mathbf{2 a}$ ', which was freshly prepared from the corresponding ethyl bromodifluoroacetate (2a) and zinc in THF. We were very pleased that staged treatment of a toluene

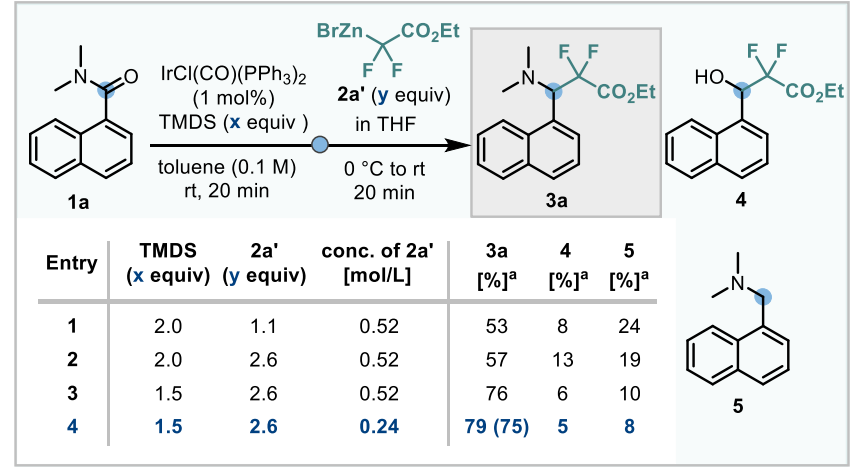

Scheme 2. Reaction optimization. ${ }^{\text {[a] }}$ NMR yield using 1,3,5-trimethoxybenzene as internal standard, isolated yield in parenthesis.

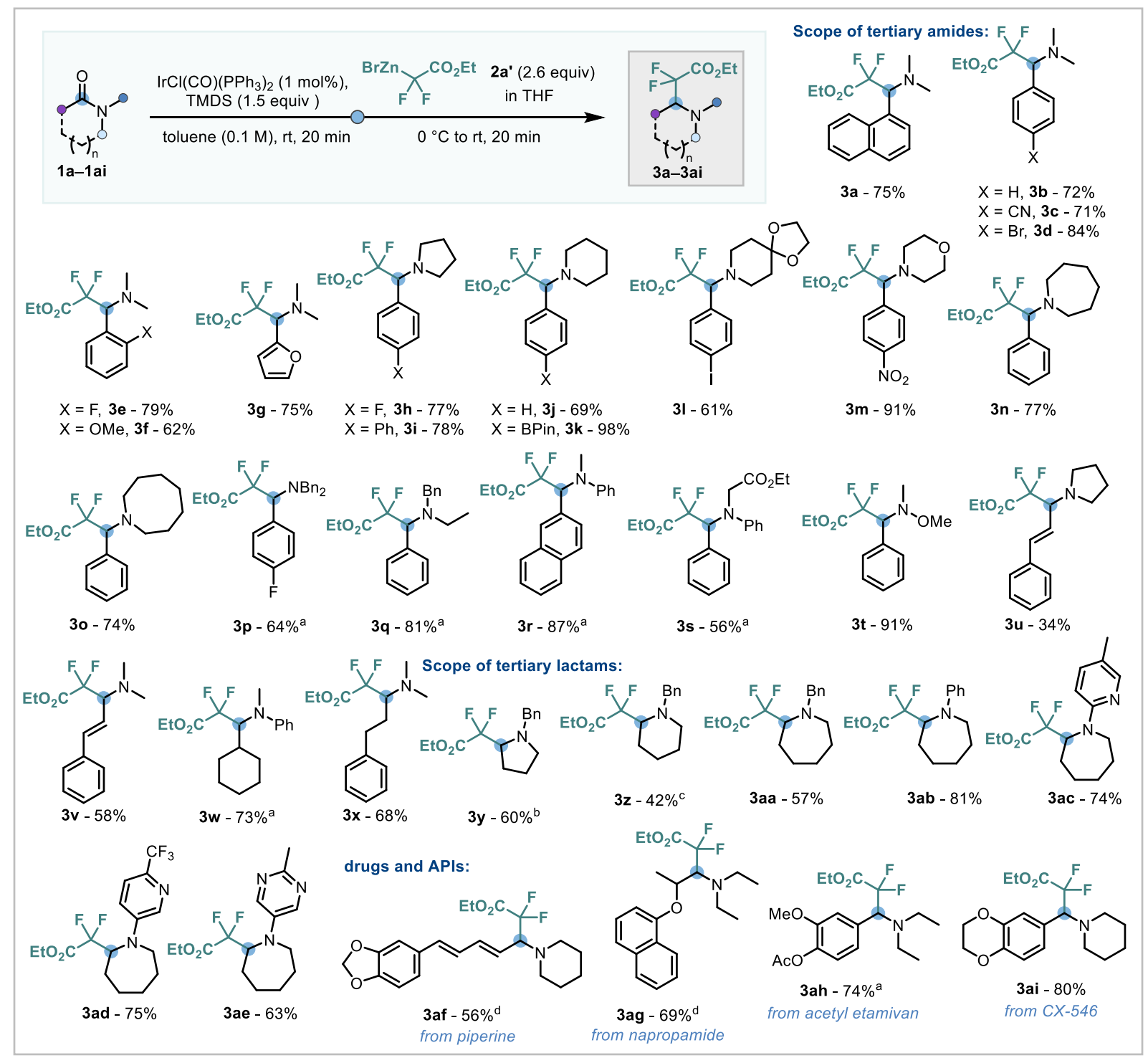

Scheme 3. Reaction scope of tertiary amides and lactams. Standard conditions: amide or lactam $1(0.15 \mathrm{mmol}), \mathrm{IrCl}(\mathrm{CO})(\mathrm{PPh}))_{2}(1 \mathrm{~mol} \%), \mathrm{TMDS}(0.23 \mathrm{mmol})$, toluene $(1.50 \mathrm{~mL}), 2 \mathbf{a}^{\prime}(0.40 \mathrm{mmol})$ in THF (1.63 mL); isolated yields are given; [a] 2.5 equiv TMDS and $2 \mathrm{~mol} \% \mathrm{IrCl}(\mathrm{CO})(\mathrm{PPh}) 2$ were used; [b] $2-\mathrm{Methyl-THF} \mathrm{was}$ used as the solvent in first step and first step was stirred for $2 \mathrm{~min} ;{ }^{[c]} \mathrm{THF}$ was used as the solvent in first step; ${ }^{[d]} 2.5$ equiv TMDS and 2 mol\% $\left.\mathrm{IrCl}_{(\mathrm{CO})(\mathrm{PPh}}\right)_{2}$ were used and first step was stirred for $1 \mathrm{~h}$. 
solution of 1a with $1 \mathrm{~mol} \%$ Vaska's complex, 2.0 equiv TMDS, followed by 1.1 equiv difluoro-organozinc reagent $2 \mathbf{a}^{\prime}$ gave the desired tertiary amine $3 \mathrm{a}$ in promising $53 \%$ yield, alongside minor amounts of secondary alcohol 4 and overreduction product 5 (Scheme 2, entry 1). Increasing the equivalents of organozinc reagent $2 a^{\prime}$ ' improved the yield of desired product $3 \mathbf{3 a}$ slightly (Scheme 2, entry 2). More significantly, lowering the amount of TMDS to 1.5 equivalents drastically reduced the rate of overreduction and allowed access to synthetically useful yields of functionalization product $\mathbf{3 a}$ (Scheme 2, entry 3). Finally, changing the concentration of organozinc reagent 2a' by dilution provided a $75 \%$ isolated yield (Scheme 2, entry 4). Further changes in the reaction conditions, such as using different solvent combinations, temperatures or reaction times did not have a positive effect on the reaction outcome (see SI for full optimization details).

With optimized conditions in hand, we then examined the reaction scope with respect to tertiary amides and lactams 1 (Scheme 3). Satisfyingly, several N,N-dimethyl-benzamides 1a$1 f$ with electron-deficient and electron-rich substituents in ortho or para positions, as well as furan substrate $\mathbf{1 g}$, could be successfully converted into the corresponding difluoromethylated tertiary amines $\mathbf{3 b} \mathbf{3} \mathbf{3 g}$ in good isolated yields (62-84\%). Pyrrolidine-, piperidine-, morpholine-, azepane- and azocanederived amides $\mathbf{3 h} \mathbf{- 3 0}$ were reductivly functionalized in good to excellent yields (69-98\%), whilst demonstating tolerance to various substituents such as boronic ester, acetal, iodo or nitro groups. $N, N$-Dibenzylamide $\mathbf{1 p}, N, N$-benzylethylamide $1 \mathbf{q}$ and anilide $1 \mathbf{r}$ were successfully employed to furnish the desired products $3 p-3 r$ in 64 to $87 \%$ yields. However, increased amounts of TMDS (2.5 equiv) and Vaska's complex ( $2 \mathrm{~mol} \%$ ) were used to force the slow reduction step of these more challenging substrates to full conversion. Anilide 1s, bearing an ethyl ester moiety, was converted into amine $3 \mathrm{~s}$ in the same way, albeit in a diminished $56 \%$ yield. Weinreb amide 1 t reacted smoothly to product $3 \mathbf{t}$ in $91 \%$ yield, while $\alpha, \beta$-unsaturated amides gave difluoro-products $3 \mathbf{u}$ and $3 \mathbf{v}$ in moderate $34 \%$ and $58 \%$ yields, which is due to competing conjugate addition. Furthermore, aliphatic amides $\mathbf{1} \mathbf{w}$ and $\mathbf{1 x}$ underwent reductive functionalization in $73 \%$ and $68 \%$ yields. Encouraged by these results, we also envisioned including lactams in the substrate scope. Five and six membered lactams $\mathbf{1 y}$ and $\mathbf{1 z}$ gave the corresponding difluoroalkylated pyrrolidine $\mathbf{3 y}$ and piperidine $\mathbf{3 z}$ in moderate $60 \%$ and $42 \%$ yields, despite slightly reoptimized reaction conditions. Very pleasingly, $\mathrm{N}$-benzyl, $\mathrm{N}$-phenyl and $\mathrm{N}$ heteroaromatic substituted difluoroalkylated azepanes 3aa-3ad were obtained in overall good yields (57-81\%) under the standard reaction conditions. This method was also successfully applied to the late stage functionalization of the active pharmaceutical ingredients (APIs) piperine (1af), napropamide (1ag), acetyletamivan (1ah) and CX-546 (1ai). The corresponding difluorinated drug derivates 3af-3ai were isolated in good yields (56-80\%), highlighting the potential application of this method for pharmaceutical drug discovery and lead structure optimization. No $\mathrm{C}-\mathrm{C}$ coupling was observed using secondary amides, and mainly aldehyde formation was witnessed after aqueous workup.

Next, we assessed the scope of the difluoro-organozinc reagents 2', and were again pleased to find that azepan-1$\mathrm{yl}($ phenyl)methanone (1n) could be readily functionalized with benzyl, trimethylsilyethyl and isopropyl difluoroacetates $\mathbf{2 b} \mathbf{2} \mathbf{- 2 d}$ ' to form 3aj-3al in good yields (63-85\%) (Scheme 4). 2 mol\% of Vaska's complex and 2.5 equiv of TMDS were used to ensure starting amide $1 \mathrm{n}$ got fully converted into the silylated hemiaminal intermediate before adding the nucleophile.

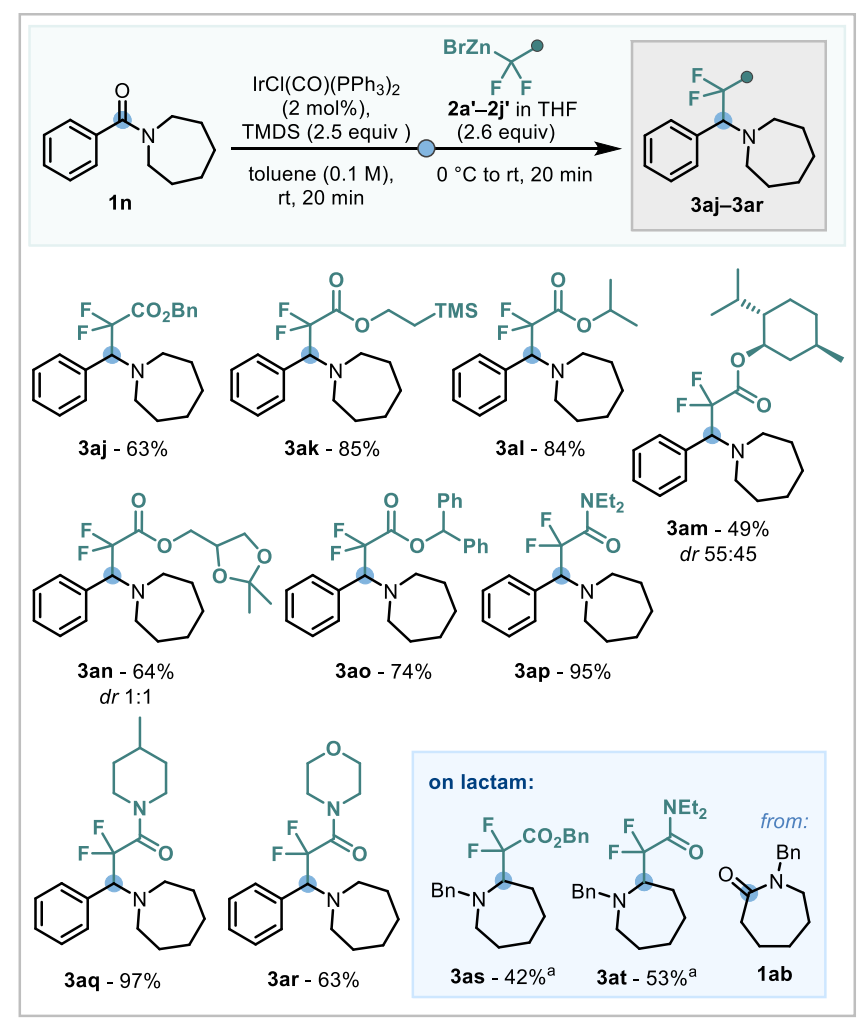

Scheme 4. Reaction scope of difluoro-organozinc reagents. Standard conditions: amide $1 \mathrm{n} \quad(0.15 \mathrm{mmol}), \quad \operatorname{IrCl}(\mathrm{CO})\left(\mathrm{PPh}_{3}\right)_{2} \quad(2 \mathrm{~mol} \%)$, TMDS (0.38 mmol), toluene $(1.50 \mathrm{~mL}), \mathbf{2}^{\prime}(0.40 \mathrm{mmol})$ in THF; isolated yields are given; [a] lactam $1 \mathrm{ab}(0.15 \mathrm{mmol}), 1.5$ equiv TMDS and $1 \mathrm{~mol} \% \operatorname{IrCl}(\mathrm{CO})\left(\mathrm{PPh}_{3}\right)_{2}$ were used.

Employing L-menthol- and glycerol-derived difluoroacetates $\mathbf{2} \mathbf{e}^{\prime}$ and 2f', products 3am and 3an were isolated in $49 \%$ and $64 \%$ yields as 1.2:1 and 1:1 mixtures of diastereomers, respectively. Sterically demanding benzhydryl difluoroacetate $\mathbf{2} \mathbf{g}$ ' could be introduced efficiently in $74 \%$ yield to give tertiary amine 3 ao. Notably, difluoroacetamide containing zinc bromides $\mathbf{2} \mathbf{h}^{\prime}$ and $\mathbf{2 i}$ could also be used under the same reaction conditions to furnish amines 3ap and 3aq in near quantitative yields. Using morpholine-derived difluoroacetamide 2j', 3ar was obtained in a good yield (63\%). Highlighting lactams as suitable feedstock compounds, the reductive functionalization of $1 \mathbf{a b}$ with benzyl difluoroacetate $\mathbf{2} \mathbf{b}$ ' and difluoroacetamide $\mathbf{2} \mathbf{h}$ ' gave the C2difluoroalkylated saturated nitrogen containing heterocyclic amines 3 as and 3 at in $42 \%$ and $53 \%$ yields.

To showcase the synthetic utility of this methodology we performed a gram scale reductive difluoroalkylation of amide $\mathbf{1 b}$, generating tertiary amine $3 \mathrm{~b}$ in a $67 \%(1.15 \mathrm{~g}, 4.47 \mathrm{mmol})$ yield (Scheme 5), which was comparable to the small-scale reaction. Identifying the ester moiety in $\mathbf{3 b}$ as a useful handle for downstream derivatizations, we synthesized several $\mathrm{CF}_{2-}$ containing compounds $\mathbf{6 - 1 0}$ by standard organic procedures. Primary alcohol 6 was obtained in $81 \%$ yield by reduction with $\mathrm{NaBH}_{4}$. Addition of a methanolic ammonia solution gave corresponding primary amide 7 in $85 \%$ yield. Tertiary alcohol 8 was formed in $61 \%$, using 2.1 equivalents of Grignard reagent. 
Saponification and subsequent acidification furnished carboxylic acid $\mathbf{9}$ in quantitative yield. Finally, enol ether $\mathbf{1 0}$ was installed in $42 \%$ by employing the Tebbe reagent under basic reaction conditions.

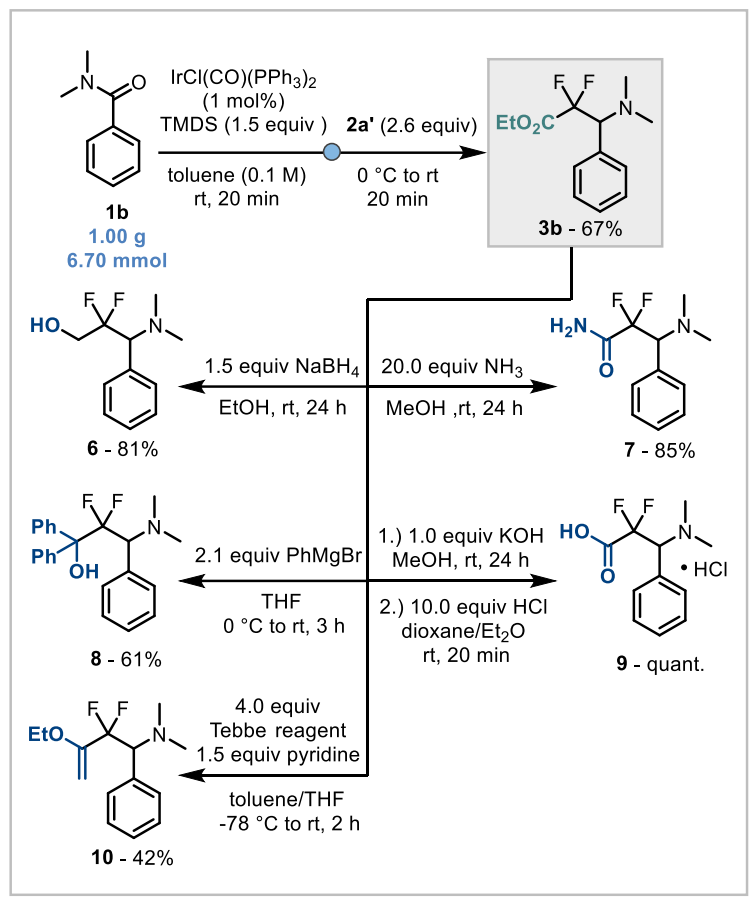

Scheme 5. Gram scale reaction and downstream functionalization. Isolated yields are given.

In conclusion, a broadly applicable and efficient method for the synthesis of acyclic and cyclic $\alpha$-difluoroalkylated tertiary amines with good overall yields, has been developed. The mild iridium-catalyzed reductive difluoroalkylation shows excellent functional group tolerance with respect to both coupling partners; amides/lactams and organozinc reagents, which is among other things highlighted by the late stage derivatization of four drug molecules. Furthermore, the reaction was readily performed on gram scale without a significant loss in yield and several $\mathrm{CF}_{2-}$ containing derivates were made by common downstream transformations, altogether demonstrating the potential utility of the method developed herein as a useful tool in current and future drug discovery programs.

\section{Acknowledgements}

This project has received funding from the European Union's Horizon 2020 research and innovation programme under the Marie Sklodowska-Curie grant agreement No 892540. The authors thank Tatiana Rogova, Daniel Matheau-Raven, Yaseen Almehmadi, Daniel Rozsar and Andrew Maitland, as well as former group members, for starting material synthesis and enriching discussions.

Keywords: difluoroalkylation • tertiary amides • Reformatsky• iridium $\cdot \mathrm{C}-\mathrm{C}$ coupling
[1] a) G. M. Blackburn, D. E. Kent, F. Kolkmann, J. Chem. Soc. Chem Commun. 1981, 1188-1190; b) G. M. Blackburn, D. E. Kent, F. Kolkmann, J. Chem. Soc. Perkin Trans. 1 1984, 1119-1125; c) W. B. Motherwell, M. J. Tozer, B. C. Ross, J. Chem. Soc. Chem. Commun. 1989, 1437-1439.

[2] a) K. Müller, C. Faeh, F. Diederich, Science 2007, 317, 1881-1886; b) S. Purser, P. R. Moore, S. Swallow, V. Gouverneur, Chem. Soc. Rev. 2008, 37, 320-330; c) D. O'Hagan, Chem. Soc. Rev. 2008, 37, 308-319 d) Y. Zhu, J. Han, J. Wang, N. Shibata, M. Sodeoka, V. A. Soloshonok, J. A. S. Coelho, F. D. Toste, Chem. Rev. 2018, 118, 3887-3964; e) D. E. Yerien, S. Barata-Vallejo, A. Postigo, Chem. Eur. J. 2017, 23,1467614701 ; f) R. Britton, V. Gouverneur, J.-H. Lin, M. Meanwell, C. Ni, G. Pupo, J.-C. Xiao, J. Hu, Nat Rev Methods Primers 2021, 47, 1-22.

[3] a) Q. Peng, B. Yan, F. Li, M. Lang, B. Zhang, D. Guo, D. Bierer, J. Wang, Commun. Chem. 2021, 4, 148-155; b) M. Braun, Eur. J. Org. Chem. 2021, 1825-1836; c) O. O. Fadeyi, C. W. Lindsley Org. Lett. 2009, 11, 4 943-946; d) H.-J. Böhm, D. Banner, S. Bendels, M. Kansy, B. Kuhn, K. Müller, U. Obst-Sander, M. Stahl, ChemBioChem 2004, 5, 637-643; e) W. K. J. Hagmann, Med. Chem. 2008, 51, 4359-4369; f) P. Jeschke, ChemBioChem 2004, 5, 570-589; g) M. Morgenthaler, E. Schweizer, A. Hoffmann-Röder, F. Benini, R. E. Martin, G. Jaeschke, B. Wagner, H. Fischer, S. Bendels, D. Zimmerli, J. Schneider, F. Diederich, M. Kansy, K. Müller, ChemMedChem 2007, 2, 1100-1115.

[4] a) W. Plunkett, P. Huang, Y. Z. Xu, V. Heinemann, R. Grunewald, V. Gandhi, Semin. Oncol. 1995, 22, 3-10; b) S. Noble, K. L. Goa, Drugs 1997 54, 447-472.

[5] D. Ferraris, B. Duvall, G. Delahanty, B. Mistry, J. Alt, C. Rojas, C Rowbottom, K. Sanders, E. Schuck, K.-C. Huang, S. Redkar, B. B Slusher, T. Tsukamoto, J. Med. Chem. 2014, 57, 6, 2582-2588.

[6] J. A. B. Balfour, K. McClellan, Am. J. Clin. Dermatol. 2001, 2, 197-201.

[7] R. Hong, K. Edgar, K. Song, S. Steven, A. Young, P. Hamilton, A Arrazate, C. De La Cruz, C. Chan, J. Pang, L. Salphati, M. Belvin, M. Nannini, S. Staben, L. Friedman, D. Sampath, Cancer Res. 2018, 78, (4 Suppl): Abstract nr PD 4-14.

[8] K. A. Salam, N. Akimitsu, BioMed Research International 2013, Article ID 467869, 1-9.

[9] J. B. I. Sap, C. F. Meyer, N. J. W. Straathof, N. Iwumene, C. W. am Ende, A. A. Trabanco, V. Gouverneur, Chem. Soc. Rev. 2021, 50, 8214-8247.

[10] a) P. J. Czerwinski, B. Furman Front. Chem. 2021, 9, 148-157; b) D. Matheau-Raven, P. Gabriel, J. A. Leitch, Y. A. Almehmadi, K. Yamazaki, D. J. Dixon, ACS Catal. 2020, 10, 15, 8880-8897; c) D. Y. Ong, J.-H. Chen, S. Chiba, Bull. Chem. Soc. Jpn. 2020, 93, 1339-1349.

[11] a) P.-Q. Huang, Y.-H. Huang, K.-J. Xiao, Y. Wang, X.-E. Xia, J. Org. Chem. 2015, 80, 2861-2868; b) K.-J. Xiao, A.-E. Wang, P.-Q. Huang, Angew. Chem. Int. Ed. 2012, 51, 8314-8317; c) K.-J. Xiao, J.-M. Luo, K.Y. Ye, Y. Wang, P.-Q. Huang, Angew. Chem. Int. Ed. 2010, 49, 30373040; d) K.-J. Xiao, Y. Wang, K.-Y. Ye, P.-Q. Huang, Chem. Eur. J. 2010, 16, 12792-12796.

[12] a) K. Shirokane, T. Wada, M. Yoritake, R. Minamikawa, N. Takayama, T. Sato, N. Chida, Angew. Chem. Int. Ed. 2014, 53, 512-516; b) G. Vincent, R. Guillot, C. Kouklovsky, Angew. Chem. Int. Ed. 2011, 50, 1350-1353; c) K. Shirokane, Y. Kurosaki, T. Sato, N. Chida, Angew. Chem. Int. Ed. 2010, 49, 6369-6372; d) M. Nakajima, Y. Oda, T. Wada, R. Minamikawa, K. Shirokane, T. Sato, N. Chida, Chem. Eur. J. 2014, 20, 17565-17571 e) T. Sato, N. Chida, Org. Biomol. Chem. 2014, 12, 3147-3150; f) Y. Oda, T. Sato, N. Chida, Org. Lett. 2012, 14, 950-953.

[13] D. Y. Ong, D. Fan, D. J. Dixon, S. Chiba, Angew. Chem. Int. Ed. 2020 59, 11903-11907.

[14] a) P. Gabriel, A. W. Gregory, D. J. Dixon, Org. Lett. 2019, 21, 66586662; b) L.-G. Xie, D. J. Dixon, Nat. Commun. 2018, 9, 2841-2849; c) Á L. Fuentes de Arriba, E. Lenci, M. Sonawane, O. Formery, D. J. Dixon, Angew. Chem. Int. Ed. 2017, 56, 3655-3659; d) P. W. Tan, J. Seayad, D. J. Dixon, Angew. Chem. Int. Ed. 2016, 55, 13436-13638; e) A. W. Gregory, A. Chambers, A. Hawkins, P. Jakubec, D. J. Dixon, Chem. Eur. J. 2015, 21, 111-114; f) T. Rogova, P. Gabriel, S. Zavitsanou, J. A. Leitch, F. Duarte, D. J. Dixon, ACS Catal. 2020, 10, 11438-11447; g) K. Yamazaki, P. Gabriel, G. Di Carmine, J. Pedroni, M. Farizyan, T. A. Hamlin, D. J. Dixon, ACS Catal. 2021, 11, 7489-7497; h) D. MatheauRaven, D. J. Dixon, Angew. Chem. Int. Ed. 2021, 60, 19725-19729; i) P. Gabriel, Y. A. Almehmadi, Z. R. Wong, D. J. Dixon, J. Am. Chem. Soc 
2021, 143, 29, 10828-10835; j) P. Gabriel, L.-G. Xie, D. J. Dixon, Org. Synth. 2019, 96, 511-527; k) L.-G. Xie, D. J. Dixon, Chem. Sci. 2017, 8 7492-7497.

[15] a) Y. Takahashi, T. Sato, N. Chida, Chem. Lett. 2019, 48, 1138-1141; b) M. Yoritate, Y. Takahashi, H. Tajima, C. Ogihara, T. Yokoyama, Y. Soda T. Oishi, T. Sato, N. Chida, J. Am. Chem. Soc. 2017, 139, 18386-18391 c) S. Katahara, S. Kobayashi, K. Fujita, T. Matsumoto, T. Sato, N. Chida, J. Am. Chem. Soc. 2016, 138, 5246-5249; d) M. Nakajima, T. Sato, N. Chida, Org. Lett. 2015, 17, 1696-1699; e) Y. Soda, Y. Sugiyama, M. Yoritate, H. Tajima, K. Shibuya, C. Ogihara, T. Oishi, T. Sato, N. Chida Org. Lett. 2020, 22, 7502-7507; f) S. Katahara, Y. Sugiyama, M. Yamane, Y. Komiya, T. Sato, N. Chida, Org. Lett. 2021, 23, 3058-3063 g) W. Ou, F. Han, X.-H. Hu, H. Chen, P.-Q. Huang, Angew. Chem. Int Ed. 2018, 57, 11354-11358; h) P.-Q. Huang, W. Ou, F. Han, Chem. Commun. 2016, 52, 11967-11970.

[16] a) S. Mizuta, I. S. R. Stenhagen, M. O'Duill, J. Wolstenhulme, A. K. Kirjavainen, S. J. Forsback, M. Tredwell, G. Sandford, P. R. Moore, M. Huiban, S. K. Luthra, J. Passchier, O. Solin, V. Gouverneur, Org. Lett. 2013, 15, 2648-2651; b) W. J. Middleton, E. M. Bingham, J. Org. Chem. 1980, 45, 2883-2887; c) Y.-Y. Yang, J. Xu, Z.-W. You, X.-H. Xu, X.-L. Qiu, F.-L. Qing, Org. Lett. 2007, 9, 5437- 5440; d) K. Fujikawa, Y. Fujioka, A. Kobayashi, H. Amii, Org. Lett. 2011, 13, 5560-5563; e) A Kobayashi, H. Amii, Synthesis 2012, 44, 3015-3018; f) T. Otsuka, A Ishii, P. A. Dub, T. Ikariya, J. Am. Chem. Soc. 2013, 135, 9600-9603; g) Q. Wang, H. Gong, Y. Zhang, Y. Peng, H. Chen, M. Li, H. Deng, J. Hao, W. Wan, Org. Biomol. Chem. 2021, 19, 7867-7874; h) O. V Geraschenko, V. V. Solomina, B. V. Vashchenkoa, P. Khodakivskyib, A A. Tolmachev, O. O. Grygorenkoa, J. Fluorine Chem. 2020, 229, 109407-109415; i) Z. Ruan, S.-K. Zhang, C. Zhu, P. N. Ruth, D.Stalke, L. Ackermann, Angew. Chem. Int. Ed. 2017, 56, 2045-2049; j) J. Jung, E. Kim, Y. You, E. J. Cho, Adv. Synth. Catal. 2014, 356, 2741-2748; k) W.-T. Fan, Y. Li, D. Wang, S.-J. Ji, Y. Zhao, J. Am. Chem. Soc. 2020 142, 20524-20530.

[17] a) M.-C. Belhomme, T. Besset, T. Poisson, X. Pannecoucke, Chem. Eur. J. 2015, 21, 12836-12865; b) Z. Feng, Y.-L. Xiao, X. Zhang, Acc. Chem. Res. 2018, 51, 2264-2278.

[18] a) D.-Q. Dong, H. Yang, J.-L. Shi, W.-J. Si, Z.-L. Wang, X.-M. Xub, Org Chem. Front. 2020, 7, 2538-2575; b) C.-H. Qu, G.-T. Song, J. Xu, W Yan, C.-H. Zhou, H.-Y. Li, Z.-Z. Chen, Z.-G. Xu, Org. Lett. 2019, 21, 8169-8173; c) Y.-Q. Zhu, L.-W. Hui, S.-B. Zhanga, Adv. Synth. Catal. 2021, 363, 2170-2176; d) W.-T. Fan, Y. Li, D. Wang, S.-J. Ji, Y. Zhao, J. Am. Chem. Soc. 2020, 142, 20524-20530.

[19] a) T. Besset, T. Poisson, X. Pannecoucke, Eur. J. Org. Chem. 2015, 2765-2789; b) L. Dai, Y.-Y. Xu, Z.-H. Xia, S. Ye, Org. Lett. 2020, 22 8173-8177; c) C. Xu, R. Cheng, Y.-C. Luo, M.-K. Wang, X. Zhang, Angew. Chem. Int. Ed. 2020, 59, 18741-18747; d) T. Mao, M.-J. Ma, L. Zhao, D.-P. Xue, Y. Yu, J. Gu, C.-Y. He, Chem. Commun. 2020, 56, 1815-1818; e) M. Zhou, H.-Y. Zhao, S. Zhang, Y. Zhang, X. Zhang, J. Am. Chem. Soc. 2020, 142, 18191-18199; f) Z. Zhang, X. Li, D. Shi, Adv. Synth. Catal. 2021, 363, 3348-3353; g) M. Zhang, J.-H. Lin, C.-M. Jin J.-C. Xiao, Chem. Commun. 2021, 57, 2649-2652; h) X. Li, S. He, Q. Song, Org. Lett. 2021, 23, 2994-2999; i) X. Li, S. Hea, Q. Song, Chem. Commun. 2021, 57, 6035-6038; j) X. Cheng, X. Liu, S. Wang, Y. Hu, B. Hu, A. Lei, J. Li, Nat. Commun. 2021, 12, 4366-4376.

[20] a) A. Otaka, H. Watanabe, E. Mitsuyama, A. Yukimasa, H. Tamamura, N. Fujii, Tetrahedron Lett. 2001, 42, 285-287; b) W. Peng, P. He, S. Zhu, Z. Li, Tetrahedron Lett. 2004, 45, 3677-3680; c) R. Pedrosa, S Sayaleroy, M. Vicente, Tetrahedron 2006, 62, 10400-10407; d) J. M. Andrés, M. A. Martínez, R. Pedrosa, A. Pérez-Encabo, Synthesis 1996, 9, 1070-1072; e) A. Vidal, A. Nefzi, R. A. Houghten, J. Org. Chem. 2001, 66, 8268-8272; f) A. Sorochinsky, N. Voloshin, A. Markovsky, M. Belik, N. Yasuda, H. Uekusa, T. Ono, D. O. Berbasov, V. A. Soloshonok, J. Org. Chem. 2003, 68, 7448-7454; g) T. L. March, M. R. Johnston, P. J. Duggan, Org. Lett. 2012, 14 (1), 182-185; h) A. Tarui, T. Ikebata, K. Sato, M. Omote, A. Ando, Org. Biomol. Chem. 2014, 12, 6484-6849; i) M. Mamone, E. Morvan, T. Milcent, S. Ongeri, B. Crousse, J. Org. Chem. 2015, 80, 1964-1971. 
